\title{
Gircadian Rhythm of Electrical Activity and Motility of the Stomach in Cats and Their Relation to Sleep-Wakefulness Stages
}

\author{
Yoshiko Fujitani and Masae Hosogai \\ Department of Physiology, Tottori University School of \\ Medicine, Yonago 683
}

\begin{abstract}
Fujitani, Y. and Hosogai, M. Circadian Rhythm of Electrical Activity and Motility of the Stomach in Cats and Their Relation to Sleep-Wakefulness Stages. Tohoku J. exp. Med., 1983, 141 (3), 275-286 — Polysomnographic recordings were done over continuous 3 days together with those of electrical and mechanical activities of the stomach using chronically implanted electrodes and forcetransducer in unanesthetized and unrestrained state of 4 adult cats. Circadian rhythm of the basic electric rhythm (BER) and mechanical activity in the stomach accustomed to feeding schedule of once a day was divided into the two; the fed state from the end of feeding to 8 to $11 \mathrm{hr}$ later and the succeeding fasting state. However, differentiation of the fasting state into the contractile and uncontractile periods, which is known in dogs, was not evident in cats. The mean BER interval was longer and the standard deviation was smaller in the fed state than in the fasting state. Among stages of wakefulness (W), drowsiness (D), light slow wave sleep (LS), deep slow wave sleep (DS) and REM sleep, the mean BER intervals in every total time during the fed state were compared with one another. A definite unidirectional finding obtained in all 4 cats was only that the mean BER intervals in the stage $D$ were longer significantly than those in the stage $W$. gastric activity; circadian rhythm; sleep; REM
\end{abstract}

So far, diurnal changes in motor and/or electrical activities of the stomach have been studied, using open tipped catheter in humans (Bloom et al. 1970), extraluminal force-transducer in dogs (Itoh et al. 1977; Kamihogi 1977) and in turkeys (Duke and Evanson 1976) and extraluminal electrodes in dogs (Kamihogi 1977). In cats, however, electrical activities of the stomach were recorded only in anesthetized state (Bozler 1945) and together with mechanical activities also only in isolated preparations (Bortoff and Weg 1965). Since, in this way, little has been known on gastric activities in unanesthetized and unrestrained cats, the first objective of the present study was to provide baseline data of diurnal gastric activities in cats.

Cats have been, on the other hand, subjected to the study of sleep-wakefulness cycle in animals for the most part. However, we have no similar information in cats as in dogs (Kamihogi 1977) on how rhythms of sleep and waking influence upon gastric activities. Thus, the second objective of this research was to clarify their influence mainly on the electrical activities of the stomach in cats.

Received for publication, March 17, 1983. 


\title{
Experimental Methods
}

\author{
Animals. From 4 adults cats, successful results of chronic experiments were \\ obtained.
}

Implantation of electrodes and force-transducer. Under anesthesia by intramuscular injection of pentobarbital sodium in a dose of $30 \mathrm{mg} / \mathrm{kg}$, an aseptic operation was done. Two sets of bipolar electrodes were sewed respectively into the serosal portions of the gastric antrum and duodenum. Each stainless steel wire of bipolar electrodes used was $100 \mu \mathrm{m}$ in diameter and was insulated with enamel except for its tip. The distance between both tips was $2 \mathrm{~mm}$. These electrodes were made after McCoy and Bass (1963). A water-proof strainguage force-transducer was implanted on the angular serosa of the greater curvature in such a direction as to monitor contractile activity in the circular muscles of the stomach. This force-transducer was made after Itoh (1976). Other two silver wire electrodes of $0.5 \mathrm{~mm}$ in each tip diameter for recording the electrocorticogram (ECoG) were fixed with dental cement bilaterally into the cranial bone over the temporal cortices. Bipolar recording of the ECoG was done between these bilateral temporal areas. For recording electrical activities of the dorsal hippocampus and the amygdala in either side, bipolar twisted electrodes were positioned stereotaxically (Jasper and Ajmone-Marsan 1960) and were implanted chronically. The bare diameter of stainless steel wire used for the electrodes was $200 \mu \mathrm{m}$, while the outer diameter by enamel coating was $250 \mu \mathrm{m}$. For recording the electromyogram (EMG) of the cervical muscles and the electrooculogram (EOG), bipolar silver ball electrodes were implanted respectively into the cervical muscles and bilateral temporal edges of the orbits. Wires from every electrode and a straingauge were led out at the dorsal neck through subcutaneous tissue and were connected to the polypole-connecting socket fixed to the collar of a cat.

Polysomnography and recording of gastroduodenal activities. A 12-channel polygraph (Nihon Kohden Kogyo Co., Type RM-88) was used to record electromyograms of the stomach and of the duodenum in a frequency range of 1.5 to $100 \mathrm{~Hz}$ in $-3 \mathrm{~dB}$, and ECoG, electrical activities of the hippocampus and amygdala, EMG and EOG in the respective routine frequency ranges, through pre-amplifiers (Nihon Kohden Kogyo Co., Type RB-5). For recording mechanograms of the stomach, DC amplification was used through Wheatstone bridge and a specific DC-amplifier (Nihon Kohden Kogyo Co., Type RPF-5). Its calibration was given previously as gram scale. A low-speed recorder (Yokogawa Electric Co., Type 3052) was also used to record gastric contraction curves in a slower speed $(3 \mathrm{~cm} / \mathrm{hr})$. The recording was done more than 1 week after the surgery. Every cat had been accustomed to the feeding schedule of once a day from 5:00 to 6:00 p.m. since over a month before recording.

Identification and classification of each sleep-wakefulness stage. Sleep-wakefulness stages were classified into awake (W), drowsy (D), light slow wave sleep (LS), deep slow wave sleep (DS) and REM sleep stages. Differentiation of slow wave sleep into two stages was done according to the criteria which Ursin proposed $(1968,1970)$. Drowsiness was dealt with in his paper to be included into awake stage, but it was here given a role of a single separate stage similarly as in the studies of Sterman et al. (1965) and of Lucas and Sterman (1974). The respective characteristics of each stage were as follows: The stage W-1) low voltage activity in ECoG, 2) hippocampal theta wave, 3) well sustained EMG activity, 4) some EOGs and 5) behaviorally awake. The stage $\mathrm{D}-1$ ) 4-8 $\mathrm{Hz}$ intermediate-high voltage ECoG in 2-3 sec or longer burst, 2) well sustained EMG activity, 3) occasional eye movements and 4) behavioral quiescence. The stage LS - 1) $12-14 \mathrm{~Hz}$ high voltage sleep spindles and some 1-4 Hz high voltage slow waves, both on a low voltage background, 2) variable, usually well sustained EMG activity, 3) no eye movements and 4) behavioral sleep (quiescence with closed eyes). The stage DS - 1) more than $50 \%$ of 2 inkwriting pages for 60 sec consisting of high voltage $1-4 \mathrm{~Hz}$ slow waves and $12-14 \mathrm{~Hz}$ spindles, 2) variable, but always some tonic EMG activity, 3) no eye movements and 4) behavioral sleep. The REM sleep stage - 1) low voltage fast activity in ECoG, 2) hippocampal theta waves, 3) no EMG activity, 4) bursts of rapid eye movements (REM) and 5) behavioral 
sleep. These respective stages were identified in every 1 -min record ( 2 pages of ink-writing paper) only on the third recording day atter the cat became familiar with the recording situation. The records were read by the same experimenter. The score was completed over $23 \mathrm{hr}$ except for $1 \mathrm{hr}$ of feeding time. Statistical analyses of the data were done using a minicomputer (Hitachi Co., HITAC-20) and a personal computer (Canon Co., Type BX-10).

\section{Results}

Gastric and duodenal electric potentials in the fed and fasting states

Two representative examples of electrical potentials of the stomach and duodenum and gastric contraction curve are shown in Fig. 1. Figs. 1A and B show respectively those obtained about $2 \mathrm{hr}$ after the end of feeding and about 4 $\mathrm{hr}$ before the start of feeding. Though the evidence for it will be shown later, diurnal rhythm of gastric activity in conscious cat is probably classified into the two states; the fed state and the fasting state. Thus, Figs. 1A and B correspond respectively to the figure in the fed state and that in the fasting state.

In Fig. 1A concerning the fed state, only two of gastric potential and seven of duodenal potential with almost regular but shorter intervals are seen. These regularly recurring potentials led from the walls of the stomach and duodenum in the cat are thought to be quite similar in nature to those described in the dog and other species (Daniel and Irwin 1968; Bass 1968).

\section{A. Fed state}

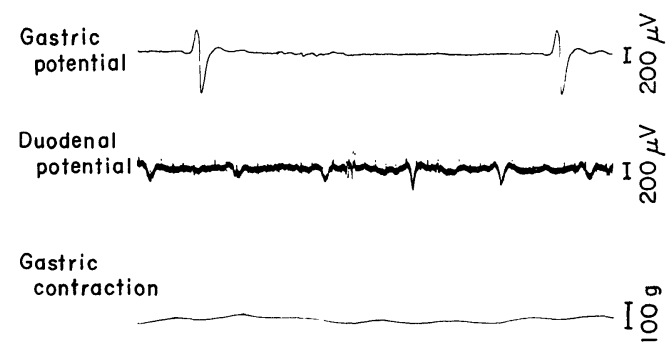

\section{B. Fasting state}

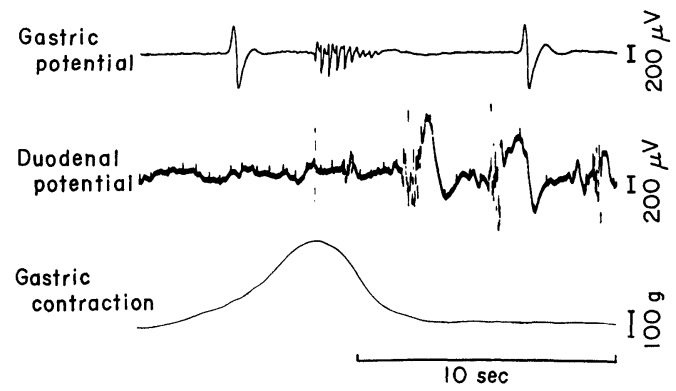

Fig. 1. Electromyograms of the stomach and of the duodenum and contraction curves of the stomach in both fed and fasting states of an unanesthetized and unrestrained cat. 
This kind of cyclical electric activity was termed the basic electric rhythm (BER) originally regarding the duodenum (Bass et al. 1961) and then the use of this term, BER, has been extended to when describing the cyclical electric activities of the overall alimentary canal and organs. Thus, the term, BER, will be used in the under-description of this paper.

In Fig. 1A, only the thirdly appearing BER of the duodenum is noticed to accompany spike potentials. Sometimes, the BER was called the initial potential while the spike potential was termed the second potential or the action potential. The reason for the use of 'action potential' is that the second potential corresponds in time to the mechanically recorded contractile activity. A fitting example for this is shown in Fig. 1B concerning the fasting state. As can be seen in the figure, the second potential which follows the first appearing BER of the stomach is accompanied by a big gastric contraction. In Fig. 1A, on the other hand, gastric contraction is not elicited in accordance with none of the gastric second potential.

Though several BERs of the duodenum in the figure are followed by the second potential, this report is not concerned with the BER and the second potential of the duodenum. Thus, the under-mentioned BER and BER interval are confined only to those of the stomach.

Further, it is noted that the gastric BER interval in the fed state is longer in time length than that in the fasting state. A quantitative analysis on this point will be described later.

\section{Diurnal rhythm of gastric activity in cats}

Fig. 2 shows an example of a continuous 24 hr's sequence of every sleepwakefulness stage, of the mean BER interval of the stomach and of its standard deviation respectively in the order of the upper, middle and lower diagrams. These results were obtained on the third recording day of a cat. These sleepwakefulness stages are the scores of every 1-min record. The means and standard deviations of the BER intervals were calculated also in every 1-min record. Around the beginning of the diagram, feeding time is shown from 5:00 to 6:00 in the evening. Then, the mean BER intervals show several rises and falls with small variations and are gradually, on the whole, decreased from the midnight to the early morning. During these temporal processes, the standard deviations of the BER intervals are continuing to be in a small range of values except for a little after the end of feeding.

From about 4:00 in the morning, other different features of the mean BER intervals are evident and are continued to be seen till the start of the next feeding time. In this new state, oscillating phenomena of the mean BER intervals with larger standard deviations are manifest. Every larger oscillating process of the mean BER intervals corresponded in phase to larger oscillating values of the standard deviations and also almost to irregular large contraction of the stomach.

In addition, it is noted that several groups of larger mean BER intervals associated with larger values of the standard deviations took place with about 1 


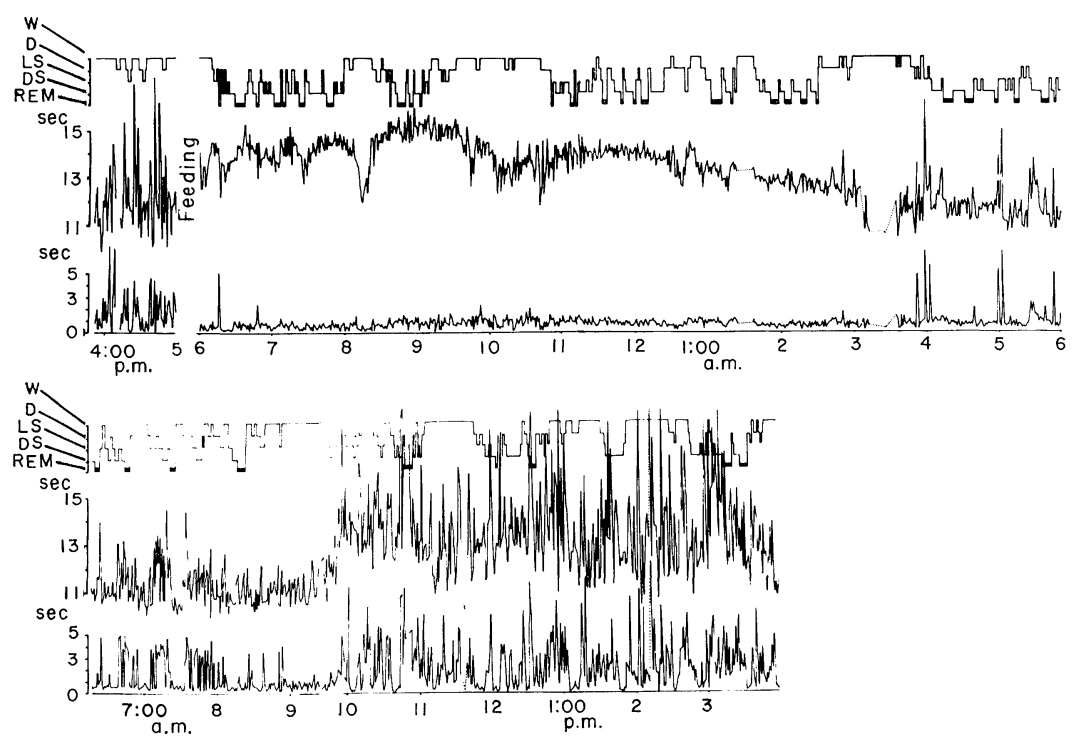

Fig. 2. Temporal sequences of the sleep-wakefulness stage, the means of gastric BER intervals and their standard deviations respectively in the upper, middle and lower diagrams. Feeding time was from 5:00 to 6:00 p.m. Recording was started from a little before 4:00 p.m. and was done for $23 \mathrm{hr}$ except for $1 \mathrm{hr}$ of feeding time.

hr interval at first and then gradually with shorter intervals. After all, oscillation of the mean BER intervals became to come out irregularly but almost continuously. The standard deviations behaved similarly.

Further, it is clearly observed from Fig. 2 that variations of the mean BER interval do not depend on the sleep-wakefulness stages. Thus, diurnal rhythm of the gastric BER interval can be divided into the two different states. The former state till 8 to $11 \mathrm{hr}$ after feeding is the fed state, while the latter one to follow the fed state and to continue till the next feeding time is the fasting state.

This classification is similar to that in dogs (Kamihogi 1977) and is found to be appropriate also from the standpoint of $23 \mathrm{hr}$ 's mechanogram of the stomach. An example is shown in Fig. 3. Since the paper speed of recording was very slow, a vertical bar represents an event of gastric contraction. In this case, the temporal processes of gastric contractions from the end of feeding time to around 5:30 in the next morning seem to be in accordance with those of the BER interval in the fed state. And, the succeeding processes for $11 \mathrm{hr}$ and $30 \mathrm{~min}$ to the start of the next feeding time are pertinent to be called those of the fasting state. Small contractions were continued to be elicited very frequently in the fed state, while there are found strong contractions with irregular but large amplitude in every several min in the fasting state. The above-mentioned findings on diurnal gastric activities were almost common to all 4 cats.

In dogs, the differentiation of the fasting state into the contractile period and the uncontractile period was noticed by several researchers (e.g., Itoh et al. 1977). 


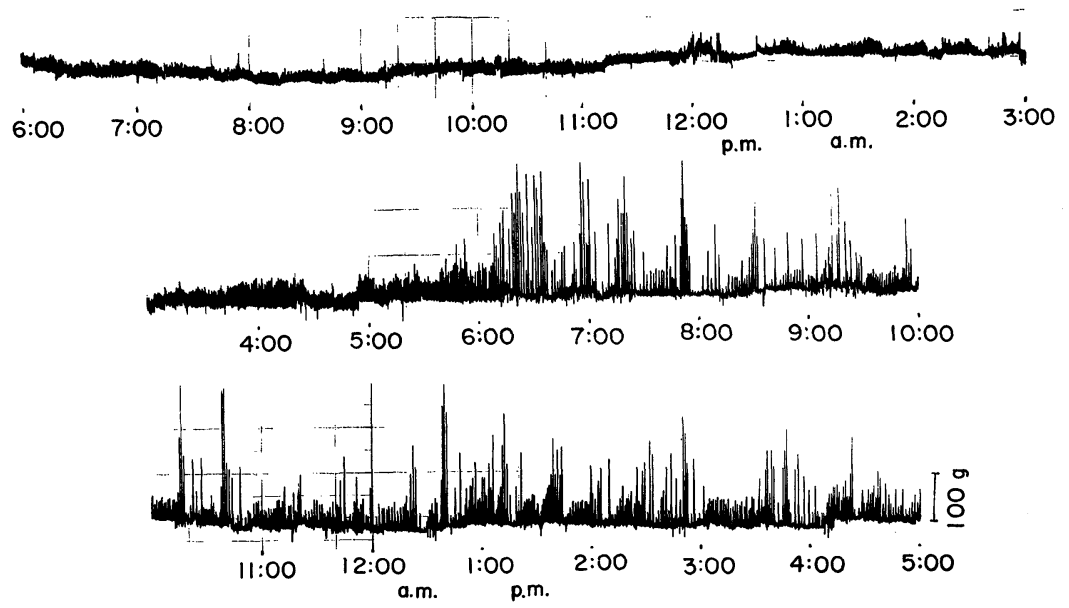

Fig. 3. An example of gastric mechanogram for $23 \mathrm{hr}$ after the end of feeding.

However, the alternation of the contractile and the uncontractile periods was not evident in the contraction curves of our all 4 cats.

Comparison of the gastric BER intervals between the fed state and the fasting state

As is shown in Fig. 1, the BER interval looks to be longer in time length in the fed state than in the fasting state. This finding was ascertained statistically in 2 cats. Table 1 shows the results obtained on the third recording day. It was deduced from $23 \mathrm{hr}$ 's polysomnographic records that the respective time lengths of the fed and fasting states were about 9 and $14 \mathrm{hr}$ in both of Cat Nos. 2 and 3. The times for which the BER interval could not be measured precisely owing to artefacts due to body movements were $1 \mathrm{hr}$ and $32 \mathrm{~min}$ in Cat No. 2 and $18 \mathrm{~min}$ in Cat No. 3.

Before examining the significance of the difference between two population means, the variance ratio test was conducted to know whether two population variances in the fed and fasting states are statistically equal or not. As a result, it was ascertained at $p<0.001$ that the standard deviations of BER intervals in

TABLE 1. Comparison of the means and standard deviations (S.D.) of BER intervals between the fed and fasting states in 2 cats (sec)

\begin{tabular}{cccc}
\hline Cat No. & Fed state & Fasting state & Total \\
\hline 2 & $13.7 \pm 0.7$ & $12.3 \pm 2.3$ & $12.8 \pm 1.8$ \\
& $(N=2299)$ & $(N=3735)$ & $(N=6034)$ \\
3 & $13.0 \pm 0.5$ & $12.2 \pm 2.0$ & $12.5 \pm 1.6$ \\
& $(N=2470)$ & $(N=3948)$ & $(N=6418)$ \\
\hline
\end{tabular}

Significant differences of the mean and the s.D. at $p<0.001$ between in the fed and fasting states of both cats. 
the fed state are smaller than those in the fasting state of both cats. This is a statistical verification of such findings in Fig. 2 that the BER intervals are more stable in the fed state than in the fasted one.

It is known that equality of population variances is not a necessary assumption when the $z$ statistic is used (Daniel 1978). Thus, the respective mean BER intervals in the fed state of 2 cats, i.e. $13.7 \mathrm{sec}$ in Cat No. 2 and $13.0 \mathrm{sec}$ in Cat No. 3, are longer respectively at $p<0.001$ than those in the fasting state, i.e. $12.3 \mathrm{sec}$ in Cat No. 2 and 12.2 sec in Cat No. 3.

Comparisons of the mean gastric BER intervals among sleep-wakefulness stages in the fed state of 4 cats

An example of the records from which the data for the comparisons were obtained is shown in Fig. 4. The stage of sleep-wakefulness cycle in this record was judged to be the REM sleep stage from the findings of low voltage fast activity of ECoG, burst of rapid eye movements, silence of EMG activity and hippocampal theta activity. As the gastric BER, only one can be seen, but each BER interval length was measured in whole period of the fed state.

Thus, only during the fed state showing more stable BER intervals but not during the fasting state showing more variable BER intervals, the means with the standard deviations of BER intervals were calculated in every total time of the

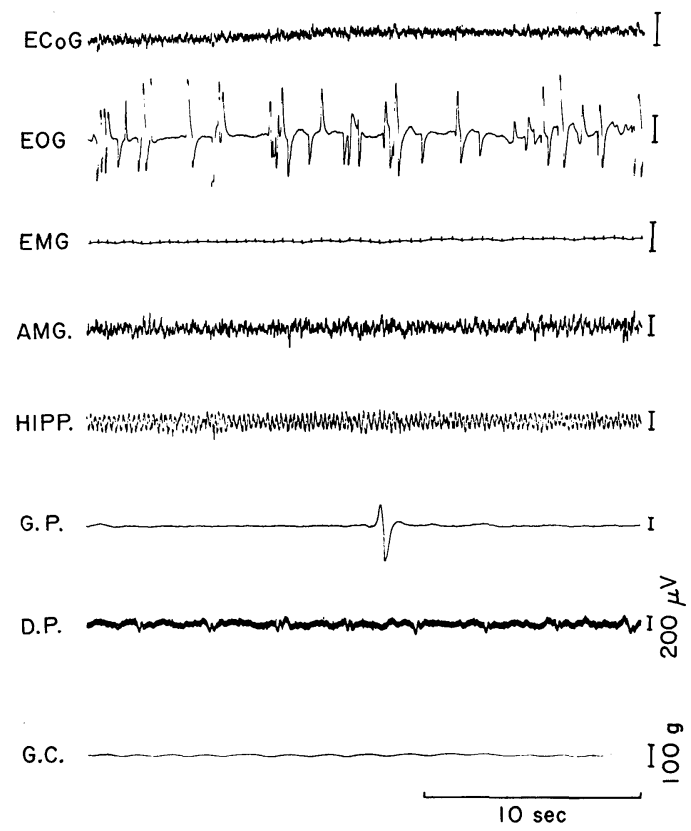

Fig. 4. An example of the records from which the sleep-wakefulness stages were respectively identified. The stage here is judged to be the REM sleep stage. AMG., amygdala; HIPP., hippocampus; G.P., gastric potential (gastric BER); D.P., duodenal potential (duodenal BER); G.C., gastric enntraction. 
sleep-wakefulness stages. The time lengths of the fed state in every cat were respectively about $9,9,11 \mathrm{hr}$ and $7 \mathrm{hr}$ and $40 \mathrm{~min}$ in Cats No. 2, No. 3, No. 6 and No. 7.

The results obtained as such are summarized in Table 2 and are diagrammed in Fig. 5. In both the table and the figure, the stages LS and DS are dealt with independently of each other and also are combined into the stage non-REM sleep

TABLE 2. Comparison of the means of total BER intervals with standard deviations (sec) during fed stste of the stomach among 5 sleep-wakefulness stages of 4 cats

\begin{tabular}{|c|c|c|c|c|c|c|c|}
\hline \multirow{2}{*}{ Cat } & \multirow{2}{*}{ Stage W } & \multirow{2}{*}{ Stage D } & \multicolumn{3}{|c|}{ Stage NREM } & \multirow{2}{*}{$\begin{array}{l}\text { Stage } \\
\text { REM }\end{array}$} & \multirow{2}{*}{ Total } \\
\hline & & & Stage LS & Stage DS & Subtotal & & \\
\hline 2 & $\begin{array}{c}13.6 \pm 0.8 \\
(530)\end{array}$ & $\begin{array}{c}13.7 \pm 0.7 \\
(509)\end{array}$ & $\begin{array}{c}13.9 \pm 0.7 \\
(298)\end{array}$ & $\begin{array}{c}13.7 \pm 0.6 \\
\quad(553)\end{array}$ & $\begin{array}{c}13.8 \pm 0.6 \\
(851)\end{array}$ & $\begin{array}{c}13.8 \pm 0.9 \\
(410)\end{array}$ & $\begin{array}{c}13.7 \pm 0.7 \\
\quad(2299)\end{array}$ \\
\hline 3 & $\begin{array}{l}12.5 \pm 0.6 \\
(298)\end{array}$ & $\begin{array}{l}13.0 \pm 0.4 \\
(893)\end{array}$ & $\begin{array}{c}13.1 \pm 0.4 \\
(418)\end{array}$ & $\begin{array}{c}13.3 \pm 0.4 \\
\quad(577)\end{array}$ & $\begin{array}{c}13.2 \pm 0.4 \\
(995)\end{array}$ & $\begin{array}{l}12.9 \pm 1.0 \\
(284)\end{array}$ & $\begin{array}{l}13.0 \pm 0.5 \\
\quad(2470)\end{array}$ \\
\hline 6 & $\begin{array}{l}11.4 \pm 1.4 \\
\quad(129)\end{array}$ & $\begin{array}{l}12.0 \pm 0.9 \\
(636)\end{array}$ & $\begin{array}{l}11.8 \pm 1.1 \\
(663)\end{array}$ & $\begin{array}{l}12.1 \pm 1.1 \\
(807)\end{array}$ & $\begin{array}{c}12.0 \pm 1.1 \\
(1470)\end{array}$ & $\begin{array}{c}12.1 \pm 0.9 \\
(488)\end{array}$ & $\begin{array}{l}11.6 \pm 1.0 \\
(2723)\end{array}$ \\
\hline 7 & $\begin{array}{c}10.9 \pm 0.9 \\
(159)\end{array}$ & $\begin{array}{c}11.2 \pm 0.9 \\
(446)\end{array}$ & $\begin{array}{c}10.9 \pm 1.0 \\
(427)\end{array}$ & $\begin{array}{c}10.8 \pm 1.4 \\
(1089)\end{array}$ & $\begin{array}{c}10.9 \pm 1.3 \\
(1516)\end{array}$ & $\begin{array}{c}10.7 \pm 1.2 \\
\quad(390)\end{array}$ & $\begin{array}{c}10.9 \pm 1.2 \\
(2511)\end{array}$ \\
\hline
\end{tabular}

W, weakefulness; D, drowsiness; NREM, non-REM sleep; LS, light slow wave sleep; DS, deep slow wave sleep, ( ), number of measurements.

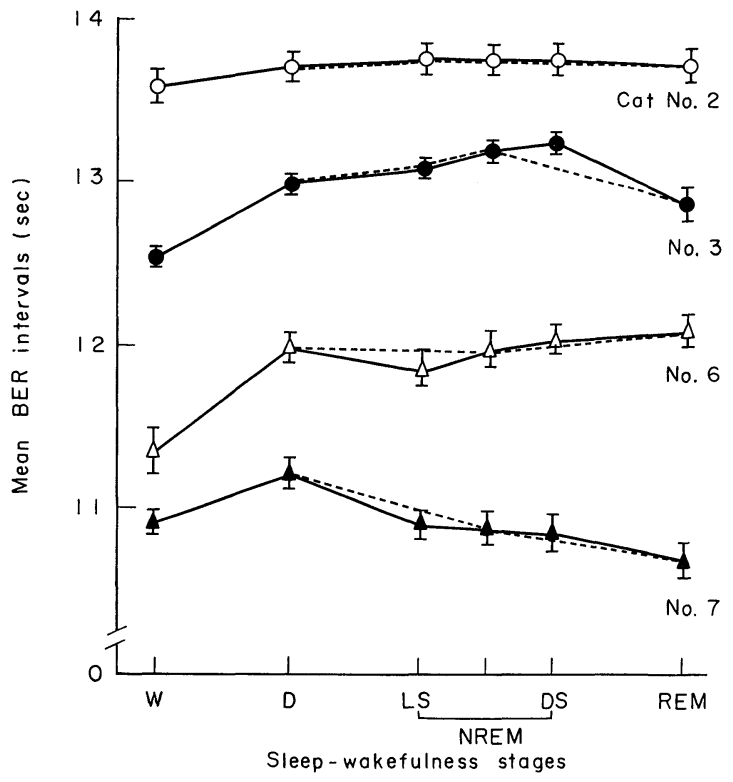

Fig. 5. Changes of the mean BER intervals with their standard deviations in total times of every sleep-wakefulness stage during the fed stage of 4 cats. Continuous lines are referred to as stages W, D, LS, DS and REM, while dotted lines are referred to as stages D, NREM which was combined from stages of LS and DS, and REM. 
(NREM). The stage $D$ is thought not to be included into stage NREM in lines of Hess et al. (1953), Sterman et al. (1965) and Lucas and Sterman (1974).

The most manifesting finding obtained from statistical analyses on these results is that the means of the gastric BER intervals in the $\mathrm{D}$ stage are longer those in the $\mathrm{W}$ stage with statistical significance $(p<0.005)$ in all 4 cats. In 2 out of 4 animals, further lengthening of the mean BER interval was significant in accompany with falling asleep (stage LS). In the other 2 animals, on the other hand, the mean BER interval was rather shortened from the stage D to the stage LS also with statistical significance. Thus, a definite conclusion will not be given on this point. Similarly in both the stage DS and the stage REM, a certain unidirectional change of the mean BER intervals was not perceived in accompany with the changes of the sleep-wakefulness stages. And no well-defined differences of the mean BER intervals and of their standard deviations could be deduced from the comparison between the stages NREM and REM.

\section{Discussion}

By applying in-vivo extraluminal force-transducer to the stomach and small intestine of dogs, Reinke et al. (1967) observed three major patterns of their contractile activities; basal, intermediate and burst. These were classification of contractile pattern, but, when describing physilogical states of the stomach and intestine, the terms of digestive and interdigestive states were used. By these researchers, all three contractile patterns were described to be present in the interdigestive state, when basal activity predominates, while no burst activity and a greater occurrence of intermediate activity compared with basal one were characteristically found in the digestive state.

Such a thought that the usage of the digestive and interdigestive states or of the fed and fasting states is sufficient for describing diurnal rhythm of gastric activity has been held also by several researchers using dogs for experimental animals (Anderson et al. 1968; Walker et al. 1974). This treatment was found to be useful for understanding circadian rhythm of the BER in dogs (Kamihogi 1977).

The present experiments confirm the existence of similar diurnal rhythm on electrical and mechamical activities of the stomach in conscious cats as that in dogs. Diurnal rhythm of gastric activities in cats is considered from both standpoints of electrical activity (BER) and motility to consist of the fed state and the fasting state. The fed state in our animals was till 8 to $11 \mathrm{hr}$ after the end of feeding of once a day. Elapsed times from the end of feeding to the start of the fasting state were $9,9,11$ and $7.6 \mathrm{hr}$ respectively in 4 cats. The succeeding state is till the start of the next feeding. Itoh et al. (1977) proposed the intervening of the intermediate state between the digestive and interdigestive states from $24 \mathrm{hr}$ 's observations of gastric motility. The time lengths of the digestive state till the initiation of the intermediate contractile pattern were 15.6, 15.9, 14.2 and $16.2 \mathrm{hr}$ respectively in their 4 dogs. Compared with these values, the time lengths of the fed state in cats seem to be shorter than those in dogs. It may be probably 
due to the differences of species, especially of the native custom of food intake.

Another difference noticed on diurnal gastric rhythm between dogs and cats, is that alternation of the contractile and uncontractile periods with fairly constant interval (77.4 min as the mean in 4 dogs, Itoh et al. 1977) was not manifested at least in gastric contrction curves of cats. There was seen, however, a small indication of alternation of a longer BER interval group to a shorter BER interval group instead of motor quiescence in Fig. 2, but this finding was not common to the other cats. The reason of no uncontractile period in cats is unknown, but it may be perhaps due to different secretory fashion of motilin which was considered to be concerned with the contractile period (e.g., Itoh et al. 1978).

In searching the literature, the studies which investigated directly the relationship between well-defined stages of sleep-wakefulness and gastric activities appeared to be very limited in number. Yaryura-Tobias et al. (1970) found in 10 human subjects who swallowed a pressure-sensitive radio capsule that higher incidence of gastric motility occurred during stages 1 and 4 than during awake stage or the other sleep stages. Stacher et al. (1975) studied gastric acid secretion during natural sleep in 4 healthy volunteers. Although there were found no significant differences between acid secretion of two sets among stages 1 to 4 and stage REM, acid secretion seemed to decrease with deeper stages of sleep. The finding obtained on dogs by Kamihogi (1977) were such that gastric BER intervals were lengthened in the transition from awake stage to NREM sleep stage only for the first half of fed state till 6 to $7 \mathrm{hr}$ after feeding.

From the above-mentioned results of previous researchers, a general inference is difficult to be deduced concerning the influences of sleep-wakefulness stages on gastric activities. However, it may be assumed that the stomach shows a general downward trend of mechanical, secretory and electrical activities in sleep compared to those in wakefulness. And also, it is interesting that neither specific nor characteristic change of gastric activities confined to the stage REM was not demonstrated. In classification of the sleep-wakefulness stages, the authors adopted the treatment to divide slow wave sleep into light slow wave sleep (LS) and deep slow wave sleep (DS) after Ursin $(1968,1970)$. Thus, the classification of awake (W), drowsy (D), LS, DS and REM sleep stages was here used. It was examined in this research what differences of mean gastric BER intervals in every total time of these stages are detected in comparing one with another of these stages. Every change of BER intervals accompanied by every alteration of sleep-wakefulness stages was not subjected with the present examination, since every stage in cats showed often a time length insufficient for statistical analysis of BER intervals.

The definite finding common to our all 4 cats was only that the mean BER interval in the stage $\mathrm{D}$ was longer than that in the stage $\mathrm{W}$. This finding seems to be in almost similar line as the result of Kamihogi. In his result, the mean BER interval was lengthened in the stage NREM from the stage W. However, the stage D cannot be considered to be included into the sleep stages (Sterman et al. 
1965; Lucas and Sterman. 1974). In fact, both further lengthening and further shortening of the mean BER intervals were observed in a comparison between the data in the stages D and LS. Thus, our finding may be substantially different from that of Kamihogi. If the stage $\mathrm{D}$ can be identified also in dogs, similar results as in cats might be obtained. The reason for these phenomena cannot be given now and further investigation will be needed for solving it.

\section{Acknowledgment}

We are grateful to Prof. T. Oikawa for his kind guidance.

\section{References}

1) Anderson, J.J., Bolt, R.J., Ulmann, B.M. \& Bass, P. (1968) Differential response to various stimulants in the body and antrum of the canine stomach. Amer. J. dig. Dis., 13, $147-156$.

2) Bass, P. (1968) In vivo electrical activity of the small bowel. In: Alimentary Canal, edited by C.F. Code, Handbook of Physiology, Section 6, Vol. IV, Amer. Physiol. Soc., Washington, D.C., pp. 2051-2074.

3) Bass, P., Code, C.F. \& Lambert, E.H. (1961) Motor and electric activity of the duodenum. Amer. J. Physiol., 201, 287-291.

4) Bloom, P.B., Filion, R.D.L., Stunkard, A.J., Fox, S. \& Stellar, E. (1970) Gastric and duodenal motility, food intake and hunger measured in man during a 24-hr period.

5) Bortoff, A. \& Weg, N. (1965) Transmission of electrical activity through the gastroduodenal junction. Amer. J. Physiol., 208, 531-536.

6) Bozler, E. (1945) The action potentials of the stomach. Amer. J. Physiol., 144, 693-700.

7) Daniel, E.E. \& Irwin, J. (1968) Electrical activity of gastric musculature. In: Alimentary Canal, edited by C.F. Code, Handbook of Physiology, Section 6, Vol. IV, Amer. Physiol. Soc., Washington, D.C., pp. 1969-1984.

8) Daniel, W.W. (1978) Biostatistics: A Foundation for Analysis in the Health Sciences. 2nd ed., John Wiley \& Sons, New York, pp. 178-179, pp. 194-196.

9) Duke, G.E. \& Evanson, O.A. (1976) Diurnal cycles of gastric motility in normal and fasted turkeys. Poult Science, 55, 1802-1807.

10) Hess, R., Koella, W.P. \& Akert, K. (1953) Cortical and subcortical recordings in natural and artificially induced sleep in cats. Electroenceph. clin. Neurophysiol., 5, $75-90$.

11) Itoh, Z. (1976) An extraluminal strain gage force transducer: its construction and implantation. Jap. J. Smooth Muscle Res., 13, 33-43. (Japanese)

12) Itoh, Z., Aizawa, I., Takeuchi, S. \& Takayanagi, R. (1977) Diurnal changes in gastric motor activity in conscious dogs. Amer. J. dig. Dis., 22, 117-124.

13) Itoh, Z., Takeuchi, S., Aizawa, L., Mori, K., Taninato, T., Seino, Y., Imura, H. \& Yanaihara, N. (1978) Changes in plasma motilin concentration and gastrointestinal contractile activity in conscious dogs. Amer. J. dig. Dis., 23, 929-935.

14) Jasper, H.H. \& Ajmone-Marsan, C. (1960) A Stereotaxic Atlas of the Diencephalon of the Cat. 1st ed., National Research Council of Canada, Ottawa.

15) Kamihogi, Y. (1977) Circadian rhythm in electrical activity and motility of the stomach and its relation to awake-sleep cycle in dogs. Yonago Acta med., 21, 57-75.

16) Lucas, E.A. \& Sterman, M.B. (1974) The polycyclic sleep-wake cycle in the cat: effects produced by sensorimotor rhythm conditioning. Exp. Neurol., 42, 347-368.

17) McCoy, E.J. \& Bass, P. (1963) Chronic electrical activity of gastroduodenal area: effects of food and certain catecholamines. Amer. J. Physiol., 205, 439-445.

18) Reinke, D.A., Rosenbaum, A.H. \& Benett, D.R. (1967) Patterns of dog gastrointestinal contractile activity monitored in vivo with extraluminal force transducers. Amer.J. dig. Dis., 12, 113-141. 
19) Stacher, G., Presslich, B. \& Stärker, H. (1975) Gastric acid secretion and sleep stages during natural night sleep. Gastroenterology, 68, 1449-1455.

20) Sterman, M.B., Knauss, T., Lehmann, D. \& Clemente, C.D. (1965) Circadian sleep and waking patterns in the laboratory cat. Electroenceph. clin. Neurophysiol., 19, 509517.

21) Ursin, R. (1968) The two stages of slow wave sleep in the cat and their relation to REM sleep. Brain Res., 11, 347-356.

22) Ursin, R. (1970) Sleep stage relations within the sleep cycles in the cat. Brain Res., 20, 91-97.

23) Walker, G.D., Stewart, J.J. \& Bass, P. (1974) The effect of parietal cell and truncal vagotomy on gastric and duodenal contractile activity of the unanesthetized dog. Ann. Surg., 179, 853-858.

24) Yaryura-Tobias, J.A., Hutcheson, J.S. \& White, L. (1970) Relationship between stages of sleep and gastric motility. Behavioral Neuropsychiatry, 2, 22-24. 